here to consume meat which is frozen at some stage increases its safety.

H WILLIAMS

KathleEN A B Williams A W L Joss

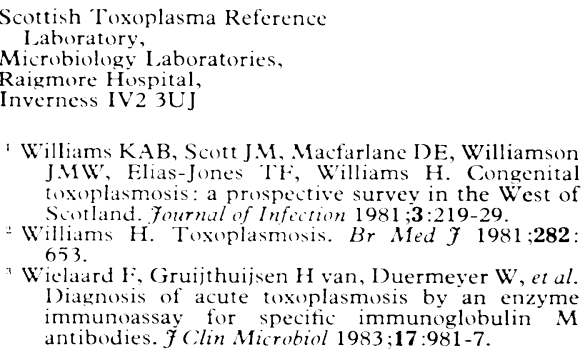

\section{Oral rehydration}

SIR,-The clinical range of diarrhoeal disease extends from a trivial and transient inconvenience to a dangerous illness which dehydrates the body, depletes it of essential electrolytes, precipitates acidosis, and may lead to death. Unfortunately, at the outset parents cannot tell if their child's symptoms are the start of a limited or a life threatening episode. Definite evidence exists that oral rehydration as the immediate response of the family to diarrhoea decreases the severity of the illness and even reduces the death rate. ${ }^{1}$

Dr Michael Watkinson (27 August, p 618) rightly points out that to wait for a doctor's prescription for an oral rehydration powder causes delay at the very time when early intervention could be most beneficial and that buying proprietary rehydration mixtures over the counter is costly. He emphasises the usefulness of a special plastic measuring spoon to enable parents to make up a scientifically authenticated sugar-salt solution. ${ }^{2}$ (Such spoons are available in the United Kingdom with instructions in several languages from TALC (Teaching Aids at Low Cost), Institute of Child Health, 30 Guilford Street, London WC1N 1EM.) There is, however, no need to wait for either a prescription or a plastic spoon. At the first signs of diarrhoea any clear fluid acceptable to the child should be given at frequent intervals even if there is also some vomiting. Carrot, rice, or chicken soup are traditional in some communities and herbal teas or fruit juices in others. Barley water and arrowroot have their advocates, and the concept behind some of these remedies is being reinforced by the recent findings that another starch based mixture, rice powder with electrolytes, is effective for rehydration. ${ }^{3}$ More severe diarrhoea with some signs of dehydration or acidosis certainly does better with a more complete formula like oral rehydration salts.

Every country needs to identify what is the most appropriate and acceptable first aid treatment for diarrhoea. In the United Kingdom the most available and efficacious home remedy is a sugar and salt mixture measured with the most available tool, the teaspoon. One level $5 \mathrm{ml}$ teaspoonful of salt and 8 level teaspoonfuls of sugar in one litre of water provide a mixture that utilises the linked sodium glucose-sucrose transport mechanism. (In these days of various shapes and sizes in teaspoons, when in doubt use the smallest size.) It is difficult to overhydrate orally, but a rough guide to quantity is to give a larger child $200 \mathrm{ml}$ (a glassful) of the fluid for every diarrhoeal stool passed, and half that amount for a small child.

Our conversations with local pharmacists suggest that many of them would welcome some kind of leaflet containing these instructions being available in this country with clear indications when professional advice needs to be sought. Perhaps this could also be distributed by infant welfare clinics and health visitors. Diarrhoea Dialogue is a quarterly newsletter which exists to disseminate just this sort of information worldwide to a whole range of professional and lay people. (It is published by the Appropriate Health Resources and Technologies Action Group, 85 Marylebone High Street, London W1M 3DE, and is sent free to anyone interested.)

There is great international concern about the five million children who die unnecessarily each year from acute diarrhoea. The need to extend communication about oral rehydration at home in all countries is urgent.

William A M Cutting

Department of Child Life and

Health

Edinburgh University,

London SW7 4Q7

Katherine M Elliott

Rahaman MM, Aziz KMS, Munshi MH, Patwari Y, Rahman M. Diarrhoeal mortality in two Bangladesh villages with and without community-based oral rehydration therapy. Lancet 1979;ii:809-12.

Clements ML, Levine MM. Cleaves F, et al. Comparison of simple sugar salt versus glucose electrolyte oral rehydration solutions in infant

Patra FC, Mahalanabis I, Jalan KN, Sen A, Baneriee givese oral rice electrolyte solution superior to Arch Dis Child 1982:57:910-2.

\section{Adjuvant chemotherapy for early breast cancer}

SIR,-While we cannot disagree with $\mathrm{Dr}$ Ian E Smith's conclusion (6 August, p 379) that current drugs for adjuvant treatment of breast cancer are not good enough, we nevertheless believe that the remainder of his review does not support his contention that treatment with the best available drugs is unjustified. It is unfortunate that negative results of a few poorly designed studies were emphasised, ${ }^{1-4}$ while a number of studies which have shown a favourable influence of intensive combination chemotherapy on disease free and overall survival were dismissed summarily. ${ }^{5-8}$ The fact that these studies were conducted in a non-randomised manner does not totally negate their results, which showed significantly lengthened survival for treated patients when compared with the untreated course of this disease. In a study which included a large population of patients with operable breast cancer treated with local modalities from several institutions results clearly showed that disease free and overall survivals of comparably staged patients were similar among institutions and to those in a group of patients used as controls for evaluating adjuvant therapy at our institution. ${ }^{9}$

The argument given for withholding chemotherapy until evidence of relapse is naive at best. Combination chemotherapy in advanced breast cancer has not resulted in the salvage of significant proportions of patients, despite the $15-20 \%$ complete remission rate achieved; most patients who achieve complete remission develop progressive disease within two years. ${ }^{1011}$ On the basis of experimental data and clinical results the most effective drugs should be used when the tumour burden is smallest in order to have the greatest likelihood of eradicating micrometastases (Schabel FM, 13th International Congress of Chemotherapy, 1983). ${ }^{12}$ It is inconsistent with experimental data to suggest that marginally effective drugs like tamoxifen or cyclophosphamide alone would have a significant impact on this disease.

AMAN U BUZDAR TERRY SMITH GeORge R BLUMENSCHEIN

University of Texas System Cancer Center,

Houston, Texas 77030

Fisher B, Ravdin RG, Ausman RK, Slack NH, Moore GE, Noer RJ. Surgical adjuvant chemotherapy in cancer of the breast: results of a decade of cooperative investigation. Ann Surg 1968;

168:337.56.
Fisher B, Carbone P, Economou SG, et al. 1-PhenyPrimary bustard (L-PAM) in the management of primary breast cancer; a report of

Rubens RD, Hayward JL, Knight RK, et al. Controlled trial of adjuvant chemotherapy with
breast cancer. Lancet $1983 ; \mathrm{i}: 839-43$. issen-Meyer R, Kjellgren K, Mansson B. Adjuvant chemotherapy in breast uzdar A, Smith T, Blumenschein G, et al. Adjuvant chemotherapy with fluorouracil, doxorubicin and cyclophosphamide (FAC) for stage II or III breast cancer: 5 year results. In: Salmon S, Jones S, eds. Adjuvant therapy of cancer. III. New York: Grune and Stratton, 1981:419-26.

Jones SE, Salmon SE, Allen H, et al. Adjuvant treatment of node-positive breast cancer with Adriamycin-cyclophosphamide with or without radiation therapy: interim results of an ongoing $162-9$.

Tormey DC, Weinberg VE, Holland JF, et al. A randomized trial of tive and three drug chemotherapy and chemoimmunotherapy in women with operable node positive breast cancer. fournal of Clinical Oncology 1983;1:138-45.

Cooper RG, Holland JF, and Glidewell O. Adjuvant chemotherapy of breast cancer. Cancer 1979;44: $793-8$

- Moon TE, Jones SE, Davis SL, et al. Development of natural history data base for breast cancer. Salmon SE, Jones SE, eds. Adjuvant therapy of Cancer II

0 S S , Buzdar AU, Smith TL, et al Complete remission in metastatic breast cancer treated with combination drug therapy. Ann Int Med 1979; 91:847-52.

1 Decker DA, Ahmann DL, Basel HF, et al. Complete responders to chemotherapy in metastatic breast cancer. FAMA 1979;242:2075-9.

2 Swenerton KD, Legha SS, Smith TL, et al. Prognostic factors in metastatic breast cancer treated with combination chemotherapy. Cancer Res 1979; 39:1552-62.

SIR,-I completely agree with Dr Ian Smith that assessments of the role of adjuvant chemotherapy in breast cancer should be based entirely on the currently available evidence. The problem is that both in his original article (6 August, p 379) and his letter (24 September, p 908) Dr Smith has omitted to mention most of the recent results and has therefore made several factually inaccurate statements.

On the basis of published studies in refereed journals and standard textbooks of medical oncology Dr Smith is incorrect on the following points. It is not naive to suppose that using different combinations of currently available drugs will improve results because this is exactly what has happened. For example, an adjuvant protocol using adriamycin and cyclophosphamide has been shown to be of definite benefit and so has a randomised study comparing the three drug programme using a combination of cyclophosphamide, methotrexate, and fluorouracil(CMF) with a five drug programme consisting of CMF with the addition of vincristine and prednisolone. ${ }^{2}$ Results at four years showed that the five drug programme was superior to $\mathrm{CMF}$ in 